\title{
An evaluation of the flammability of five dental gloves
}

\author{
R. G. Chadwick,' F. M. Tulley, ${ }^{2}$
}

Objective To assess the flammability of five brands of dental procedure glove.

Design A total of ten gloves of each brand (Biogel-D, Premier Protectors, Roeko-D, Safeskin Satin Plus and Schottlander Low Allergy) underwent flammability testing. Five of these were tested as supplied (unwashed) and five following the application of the hand disinfectant Hydrex (washed). Each glove was stretched over a metal frame and the time to ignition, when exposed to a standardised butane flame, recorded. In addition, the thickness of each glove was also assessed by micrometer measurement.

Results All the gloves tested ignited in less than 2.5 seconds. Two way analysis of variance revealed significant effects of glove type $(P<0.001)$ and treatment $(P<0.05)$, together with a significant interaction of these factors $(P<0.05)$, upon the ignition time. Washing Roeko-D gloves with Hydrex significantly $(P<0.01)$ retarded the ignition time compared with those in the unwashed state. Both the glove thickness and material type appeared to be related to ignition time.

Conclusion The work presented here demonstrates the acute and varying flammability of a range of dental procedure gloves. It should serve as a reminder to those who routinely use open flames whilst gloved of the dangers of this practice.

The present practice of dentistry requires that, in order to con1 form with accepted guidelines on cross-infection control, ${ }^{1}$ procedure gloves are worn routinely whilst treating patients. Many brands of gloves are available for this purpose. These are generally made of latex or vinyl. Whilst reports upon the dermatological effects, ${ }^{2}$ glove quality, ${ }^{3}$ chemical compatibility, ${ }^{4}$ and thermal insulating effects, ${ }^{5}$ of such products exist, no study to date has examined their flammability. This is of potential significance.

Although the usage of hot air burners has been advocated ${ }^{6}$ as a safer alternative to using an open flame whilst gloved, it is the authors' experience that such safety warnings are not heeded. Dental gloves have been demonstrated to possess a degree of thermal insulation ${ }^{5}$ and wearing them therefore blunts the temperature perception of the wearer. ${ }^{5,7}$ A delay in sensing an increase in temperature may have serious consequences for the wearer if the gloves have high flammability. It was the purpose of this work to determine and compare the flammability of five dental gloves in both the unwashed and washed state.

\section{Materials and methods}

For each product a total of ten gloves underwent flammability testing; Table 1 contains the details of the gloves tested. Five gloves were

$1^{*}$ Senior Clinical Lecturer, The Dental School, Park Place, Dundee, DD1 4HN; ${ }^{2}$ Head of Instrumentation, Department of Medical Physics, Ninewells Hospital and Medical School, Dundee

${ }^{*}$ Correspondence to: Dr R G Chadwick, The Dental School, Park Place, Dundee, DD1 4 HN

REFEREED PAPER

Received 31.07.00; Accepted 09.11.00

(C) British Dental Journal 2001; 190: 561-562 tested as supplied (unwashed) and five following washing in the disinfectant Hydrex (DePuy Medical, Leeds, UK). Prior to testing, and after the application of Hydrex, the gloves in this last group were left for a total of 10 minutes to ensure complete drying of the surface.

In order to both separate the front and back surfaces and afford a standard degree of stretch the palm section of each glove was fitted over a metal frame. This consisted of three metal posts arranged as in Figure 1. The total stretch distance around the posts was $19 \mathrm{~cm}$. For each glove, flammability testing was conducted in an atmosphere substantially free from draughts, at a temperature of $22^{\circ} \mathrm{C}$ and relative humidity of $45 \%$. A standardised flame, emitted from a burner (James Heal and Co Ltd, Halifax, UK) complying with the requirements of BS $5438(1976)^{8}$ was applied horizontally perpendicular to the face of the stretched palm section of each glove sample. Prior to each test, the rate of flow of the butane gas (Camping Gaz, Saint-Genis, France) to the burner was adjusted to ensure the horizontal reach of the flame was $23 \pm 2 \mathrm{~mm}$. With the flame extinguished, the glove was moved to a marked position with the glove

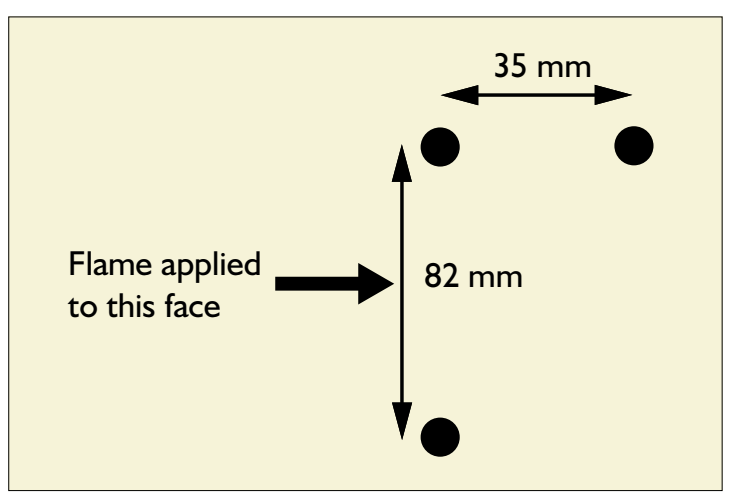

Fig. I Diagrammatic representation of the device used to stretch and hold the gloves for flammability testing as viewed from above

Table I The gloves evaluated in the study

\begin{tabular}{|c|c|c|}
\hline Glove & Manufacturer & Material \\
\hline Biogel-D & $\begin{array}{l}\text { LRC Products Ltd, } \\
\text { London, UK }\end{array}$ & $\begin{array}{l}\text { Laminate of high-tech } \\
\text { latex and hydrogel } \\
\text { polymer }\end{array}$ \\
\hline Premier Protectors & $\begin{array}{l}\text { Shermond Surgical Supply } \\
\text { Ltd, Peacehaven, UK }\end{array}$ & Vinyl \\
\hline Roeko-D & $\begin{array}{l}\text { Rexam, Langenau, } \\
\text { Germany }\end{array}$ & Nitrile \\
\hline Safeskin Satin Plus & $\begin{array}{l}\text { Safeskin Corporation, } \\
\text { San Diego, California, USA }\end{array}$ & Latex \\
\hline Schottlander Low & $\begin{array}{l}\text { Davis Schottlander and } \\
\text { Davis Ltd, Letchworth, UK }\end{array}$ & Latex \\
\hline
\end{tabular}


Table 2 The mean times in seconds and standard deviations (in brackets) to ignition recorded for the gloves tested, both unwashed and washed by application of Hydrex

\begin{tabular}{lll}
\hline Glove & Unwashed & Washed \\
\hline Biogel-D & $2.0(0.2)$ & $2.3(0.2)$ \\
Premier Protectors & $0.8(0.2)$ & $0.8(0.3)$ \\
Roeko-D & $1.1(0.2)$ & $1.6(0.2)$ \\
Safeskin Satin Plus & $1.2(0.1)$ & $1.2(0.2)$ \\
Schottlander Low Allergy & $1.8(0.2)$ & $1.6(0.2)$ \\
\hline
\end{tabular}

Table 3 The mean thickness of the gloves tested

\begin{tabular}{lcc}
\hline Glove & Mean thickness $(\mathrm{mm})$ & Standard deviation \\
\hline & & \\
Biogel-D & 0.15 & 0.02 \\
Premier Protectors & 0.06 & 0.01 \\
Roeko-D & 0.08 & 0.01 \\
Safeskin Satin Plus & 0.07 & 0.01 \\
Schottlander Low Allergy & 0.13 & 0.01 \\
\hline
\end{tabular}

face (palm section) $17 \pm 1 \mathrm{~mm}$ from the end of the burner. The flame was then ignited. From the instant that the flame was applied, the time taken for the glove to char/ignite was recorded with a stopwatch accurate to \pm 0.2 seconds. Between tests, the atmosphere of the testing-room was cleared of smoke and fumes by an extractor fan. This also ensured that the room was sufficiently oxygenated for subsequent tests. After stopping the extractor fan, adequate time for air movement to cease was both allowed and monitored by observing the steadiness of the standard flame. For each glove type and treatment sub-group the mean time and standard deviation to ignition was calculated.

In addition to the flammability test, the thickness of each glove type was measured unstretched ten times with a micrometer (961MR, Moore and Wright, Sheffield, UK) in order to determine the mean thickness of each product. The relationship of this quantity to the mean ignition time was explored by regression analysis.

\section{Results}

All the gloves tested ignited. Table 2 summarises, for each glove type and condition, the mean times to ignition and standard deviations of the observations. Two way analysis of variance revealed significant effects of glove type $(P<0.001)$ and treatment $(P<0.05)$, together with a significant interaction of these factors $(P<0.05)$, upon the ignition time. Washing Roeko-D gloves with Hydrex significantly $(P<0.01)$ retarded the ignition time compared with those in the unwashed state.

A Tukey comparison of means established that the ignition time of untreated Biogel-D gloves was significantly $(P<0.01)$ greater than for all other gloves with the exception of Schottlander Low Allergy $(P>0.05)$. Premium Protectors, Roeko-D and Safeskin Satin Plus ignited significantly $(P<0.01)$ more readily than Schottlander Low Allergy gloves. The ignition time for Safeskin Satin Plus was significantly $(P<0.05)$ greater compared with Premium Protectors but shorter compared with Biogel-D $(P<0.01)$. Roeko-D did not differ significantly $(P>0.05)$ from the ignition times observed for either Safeskin Satin Plus or Premier Protectors.

Table 3 gives the mean thickness of the gloves tested. On relating this information to the observed mean ignition times (Table 2), for both the unwashed and washed states, regression analyses demonstrated correlation coefficients of between $0.73-0.97$ for linear, exponential, logarithmic and power relationships but no single one best related these two quantities.

\section{Discussion}

Although there are currently no specific regulations relating to the flammability of surgical gloves they would appear to be covered by the General Product Safety Regulations $(1994)^{9}$ that cover the safety of daywear clothing. These require suppliers of consumer products to take steps to ensure that their products are safe under normal or reasonably foreseeable conditions of use. More stringent requirements however, relate to nightwear (Nightwear (Safety) Regulations, 1985), ${ }^{10}$ which must meet certain flammability performance requirements. Such performance is assessed using the methodology 8 adopted in this study. These regulations measure the rate of spread of flames along a material to a point 12 inches above the flame point. In order to pass, the time taken to reach this point must exceed 25 seconds. Because of the length of our samples, drawn from supplied gloves, it was not possible to make comparisons with this standard, for no glove was 12 inches long. It does however, seem unlikely that they would meet it although there is no legal requirement to do so.

All the gloves ignited and burnt rapidly in a short time (Table 2) following exposure to the flame. This was a very intense burn and would give the wearer little time to extinguish it before a serious burn resulted. Such an observation should discourage users from risking contact with a naked flame whilst gloved. The observed variation in glove flammability, from brand to brand, would appear to be related to both the material type and observed glove thickness (Table 3). In relation, however, to the flame-retardant effects of Hydrex applied to Roeko-D a satisfactory explanation is elusive. Hydrex contains both glycerol and chlorhexidene gluconate. Because of its alcohol content it would seem reasonable to expect that any trace of this product upon the glove would increase the flammability rather than retard it. It could be that for this one brand of glove one of the chemicals in Hydrex alters the surface chemistry making it more resistant to ignition. Further work is however, required to test this hypothesis.

\section{Conclusion}

The work presented here has demonstrated the acute and varying flammability of a range of dental procedure gloves. It should serve as a reminder to those who routinely use open flames whilst gloved of the dangers of this practice. In view of the risk a hot air burner, previously advocated by Brook and Lamb (1988), may offer a safer alternative.

1 British Dental Association. Advice Sheet A12 - Infection Control in Dentistry. British Dental Association, 2000.

2 Berky Z T, Luciano W J, James W D. Latex glove allergy. A survey of the US Army Dental Corps. J Am Med Assoc 1992; 268: 2695-2697.

3 Checchi L, Conti S, D'Achille C. Evaluation of the permeability of latex gloves for use in dental practice. Quint Int 1991; 22: 949-959.

4 Tinsley D, Chadwick R.G. The permeability of dental gloves following exposure to certain dental materials. J Dent 1997; 25: 65-70.

5 Chadwick R.G. The thermal insulating effects of five dental gloves. J Oral Rehabil 2000; 27: 341-343.

6 Brook I, Lamb D J. A safe alternative to the gas flame. Dent Pract 1988; 26 : 26.

7 Adoumie R, Smith B, Chiu R C J, Nohr C. Double gloving might predispose to injury by blunting temperature perception. Surgical Lapara Endo 1994; 4: 284-288.

8 BS 5438 (1976) Methods of test for flammability of vertically oriented textile fabrics and fabric assemblies subjected to a small igniting flame. London: British Standards Institution.

9 General Product Safety Regulations (1994) SI 1994/No.232. London: The Stationary Office.

10 The Nightwear (Safety) Regulations (1985) SI 1998/No.2043. London: The Stationary Office. 